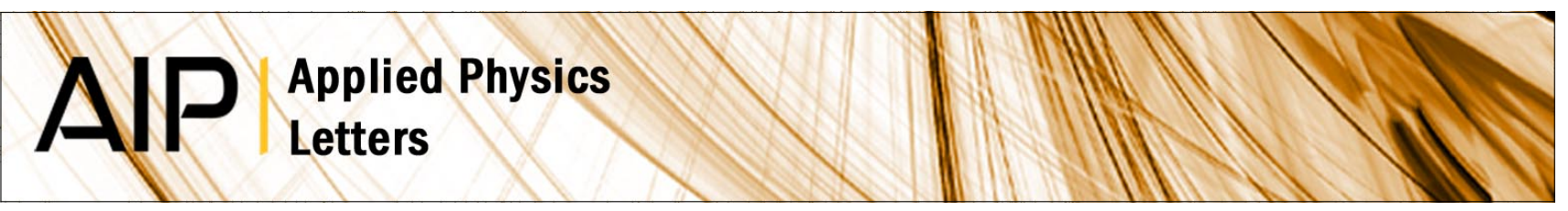

\title{
Controlling extraordinary transmission characteristics of metal hole arrays with spoof surface plasmons
}

Fumiaki Miyamaru, Mototsugu Kamijyo, Naoki Hanaoka, and Mitsuo W. Takeda

Citation: Appl. Phys. Lett. 100, 081112 (2012); doi: 10.1063/1.3689784

View online: http://dx.doi.org/10.1063/1.3689784

View Table of Contents: http://apl.aip.org/resource/1/APPLAB/v100/i8

Published by the American Institute of Physics.

\section{Related Articles}

Influence of alloy inhomogeneities on the determination by Raman scattering of composition and strain in Si1-xGex/Si(001) layers

J. Appl. Phys. 112, 023512 (2012)

Plasmon coupling in circular-hole dimers: From separation- to touching-coupling regimes

J. Appl. Phys. 112, 013113 (2012)

Correlation between reflectivity and resistivity in multi-component metallic systems

Appl. Phys. Lett. 101, 011902 (2012)

Ultrathin metallic coatings can induce quantum levitation between nanosurfaces

Appl. Phys. Lett. 100, 253104 (2012)

Transmittance and optical constants of Sr films in the 6-1220eV spectral range

J. Appl. Phys. 111, 113533 (2012)

\section{Additional information on Appl. Phys. Lett.}

Journal Homepage: http://apl.aip.org/

Journal Information: http://apl.aip.org/about/about_the_journal

Top downloads: http://apl.aip.org/features/most_downloaded

Information for Authors: http://apl.aip.org/authors

\section{ADVERTISEMENT}

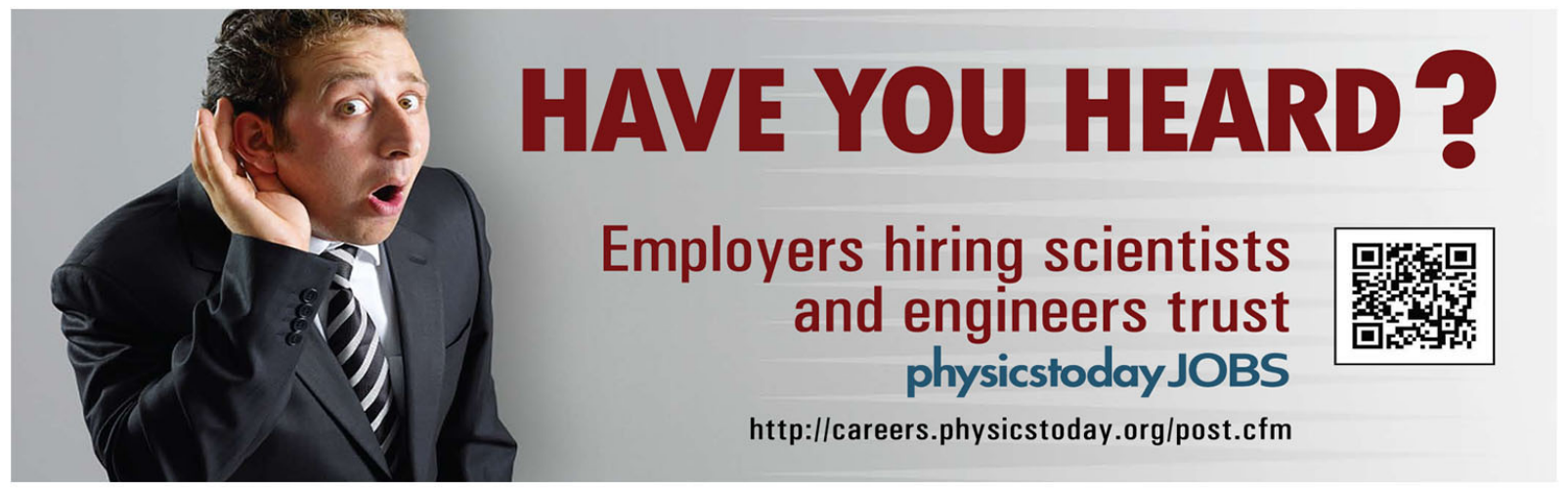




\title{
Controlling extraordinary transmission characteristics of metal hole arrays with spoof surface plasmons
}

\author{
Fumiaki Miyamaru, ${ }^{\text {a) }}$ Mototsugu Kamijyo, Naoki Hanaoka, and Mitsuo W. Takeda \\ Department of Physics, Faculty of Science, Shinshu University, 3-1-1 Asahi, Matsumoto, Nagano, Japan
}

(Received 5 January 2012; accepted 8 February 2012; published online 23 February 2012)

\begin{abstract}
We experimentally controlled the spectral characteristics of the extraordinary transmissions observed in metal hole arrays by analogy with spoof surface plasmon-polaritons (spoof SPPs). We constructed doubly periodic metal hole arrays. The larger and smaller periodic holes are for inducing extraordinary transmissions and modifying the spoof SPPs' characteristics, respectively. Introducing the smaller holes that surround the larger hole arrays can modify the dispersion curve of the surface waves excited on a structured metal surface analogous to the spoof SPP model, and consequently, the extraordinary transmission peak will show a redshift. (C) 2012 American Institute of Physics. [http://dx.doi.org/10.1063/1.3689784]
\end{abstract}

During the past few decades, many studies have examined the extraordinary transmission properties of electromagnetic waves in metal hole arrays (MHAs). ${ }^{1-7}$ In the 1960s, Ulrich et al. reported the interesting band-pass property of MHAs and attributed it to the excitation of Zenneck waves, which is now known as surface waves. ${ }^{8,9}$ Today, it is widely believed that the main mechanism responsible for extraordinary transmissions is the excitation of surface waves that originate from the interference of multiple electromagnetic waves scattered by periodically arranged holes. However, in the low-frequency region, i.e., below the terahertz $(\mathrm{THz})$ frequency region, metals are regarded as almost perfect conductors, where the localization of the surface waves near the metal surface becomes extremely weak. On the other hand, extraordinary transmissions can be seen over a broad frequency region ranging from radio frequencies to the visible regions. This fact makes it difficult to understand the common mechanism corresponding to all frequency regions. To solve this problem, Lalanne et al. ${ }^{10-12}$ proposed a cylindrical wave $(\mathrm{CW})$ model that takes into account the contribution of the surface wave. This $\mathrm{CW}$ can be excited even on a perfect conductor surface $^{12}$ and can be responsible for the extraordinary transmissions observed in the $\mathrm{THz}$ and millimeter waves, in lower-frequency regions. Later, we investigated the characteristics of this kind of surface wave in more detail by using metal hole arrays with a Kagome lattice structure and clarified the generation mechanism of the surface wave in the time domain. ${ }^{13}$ These investigations have cleared the way for providing a comprehensive explanation of the extraordinary transmission mechanism over a broad frequency range.

However, the above results also mean that the characteristics of the extraordinary transmissions cannot be modified by changing the material of metals because the absolute values of the real and imaginary parts of the permittivities of most metals are very large, and the metals can consequently be regarded as nearly perfect conductors. As a strategy to control the metal properties, Pendry proposed an effective

\footnotetext{
${ }^{\text {a) }}$ Author to whom correspondence should be addressed. Electronic mail: miyamaru@shinshu-u.ac.jp.
}

model for the periodically structured metal surface, called a spoof surface plasmon-polariton (spoof SPP) model. ${ }^{14,15}$ In this model, we can design the effective permittivity of the structured metal by introducing a sub-wavelength hole array, and consequently, the effective plasma frequency $\omega_{p}^{\text {eff }}$ comes down to the $\mathrm{THz}$, millimeter waves and microwave regions. If we fabricate the millimeter- or micrometer-scale artificial structure. In this case, the dispersion relation of the surface waves excited on the structured metal surface bends around the frequency $\omega_{p}^{e f f} / \sqrt{2}$.

So far, the dispersion relation of this spoof SPP was investigated experimentally in the microwave region by using a structured metal surface and a wire grating in reflection-type measurements. ${ }^{16}$ However, an investigation that controls the characteristics of the extraordinary transmissions of metal hole arrays by modifying the effective permittivity of the metal surface with spoof SPPs has not yet been reported. In this paper, we experimentally demonstrate how to control the spectral characteristics of the extraordinary transmissions of metal hole arrays in the $\mathrm{THz}$ region by analogy with spoof SPPs. To do so, we made doubly periodic metal hole arrays. The larger periodic holes are for inducing extraordinary transmissions, and the smaller ones are for modifying the characteristics of the spoof SPPs. When we introduced the smaller periodic holes, the extraordinary transmission peak clearly shifted to a lower frequency. We attributed this to the bend of the dispersion curve of the spoof SPP. To verify our observations, we compared the computational simulated results and the analytical results obtained from the method proposed by Garcia-Vidal et al. ${ }^{15}$ and obtained good agreement.

We fabricated through hole arrays in a square lattice on a stainless-steel film with a thickness of $20 \mu \mathrm{m}$ by using a femtosecond laser ablation process, as shown in Figs. 1 (a) -1 (c). For all samples, we fabricated square holes whose size and lattice constant are $a_{1}=250 \mu \mathrm{m}$ and $p_{1}=600 \mu \mathrm{m}$, respectively. In addition to these hole arrays (MHA1), smaller through holes were created around the aforementioned larger holes for MHA2 and MHA3 to control the characteristics of the surface waves. The lattice constants of the smaller hole array for these samples are all $150 \mu \mathrm{m}$. The hole 
(a) MHA1

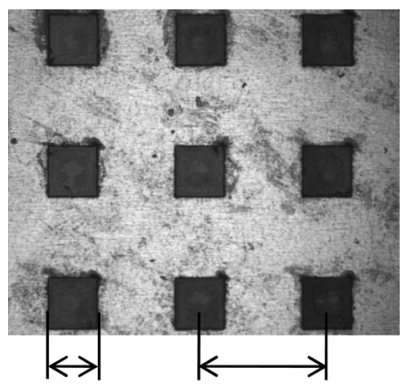

$a_{1}=250 \mu \mathrm{m} \quad p_{1}=600 \mu \mathrm{m}$ (b) MHA2

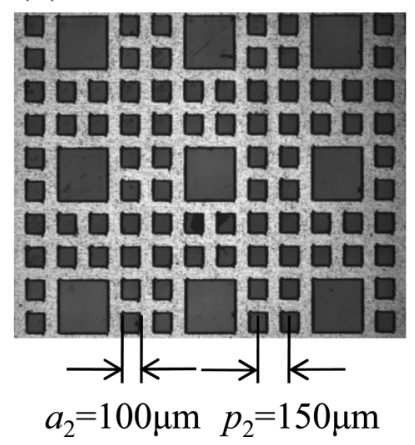

(c) MHA3

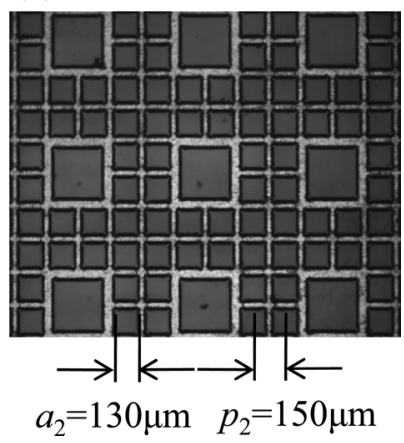

FIG. 1. Photographs of metal hole arrays used in the experiment. (a) Metal hole array (MHA1) without small hole array. (b) and (c) Metal hole arrays (MHA2 and MHA3) with small hole array. The size and lattice constant of larger holes of MHA2 and MHA3 are same to those of MHA1. sizes of the smaller holes for MHA2 and MHA3 are $100 \mu \mathrm{m}$ and $130 \mu \mathrm{m}$, respectively. We used a terahertz time-domain spectroscopy system (THz-TDS) to measure the transmission spectra of these samples. The THz-TDS allowed us to measure directly the waveform of the THz-wave in the time domain. We calculated the transmission and phase spectra via the Fourier transform of the time domain waveforms. Details of the THz-TDS system are described in our previous paper. $^{17}$

Figure 2(a) shows the measured transmission spectra of the samples at normal incidence. Each spectrum exhibits a characteristic transmission peak. The spectrum of MHA1 (black solid line) shows a transmission peak at around 0.47 $\mathrm{THz}$ with peak transmittance of $30 \%$. This transmission peak is attributed to the excitation of surface waves near the metal surface, which originate from the interference between electromagnetic waves scattered from the edges of the larger holes. ${ }^{13}$ For MHA2, a peak frequency is observed at around $0.46 \mathrm{THz}$ (red dashed line), which is shifted to the lowerfrequency side compared to that of MHA1. For MHA3, the peak frequency is further shifted and detected at $0.44 \mathrm{THz}$ (blue dot-dashed line). This observation indicates that the transmission peak is shifted to the lower-frequency side by the presence of a smaller hole array around the larger holes. The strength of the modulation of the transmission peak increases with increasing size of the smaller holes with fixed hole period. It is noted that the value of frequency shift $\delta f$ for MHA3 with respect to the peak frequency $f_{0}$ of original metal hole array (MHA1) is $\delta f / f_{0}=0.064$. The transmission intensity at the peak frequency slightly decreases by introducing the smaller holes, and a further decrease is observed with increasing size of the smaller holes. By using the finitedifference time-domain (FDTD) method, we performed a computational simulation of the samples whose geometrical parameters were the same as those in the experiment. The simulation results are shown in Figure 2(b), and they are in good agreement with the experimental results. The discrepancies in the intensities and bandwidths of the transmission peaks are attributed to the finite size effect ${ }^{4,7}$ used in the experiment. For our FDTD calculation, we modeled the unit cell of metal hole array and used periodic boundary conditions to expand the size of metal hole array infinitely. In this case, the coherent interference effect of electromagnetic waves scattered by holes becomes maximum, and correspondingly, the intensity of the transmission peak becomes high. The infinite area of MHA also makes the lifetime of the resonant oscillation of surface waves extremely long, correspondingly the bandwidth of the transmission peak becomes narrow. On the other hand, the size of area illuminated by the $\mathrm{THz}$ wave in our experiment is about $10 \mathrm{~mm}$. In this case, the aforementioned interference effect becomes weaker, and also the lifetime of surface waves becomes shorter. As the result, the intensities of the transmission peak

(a)

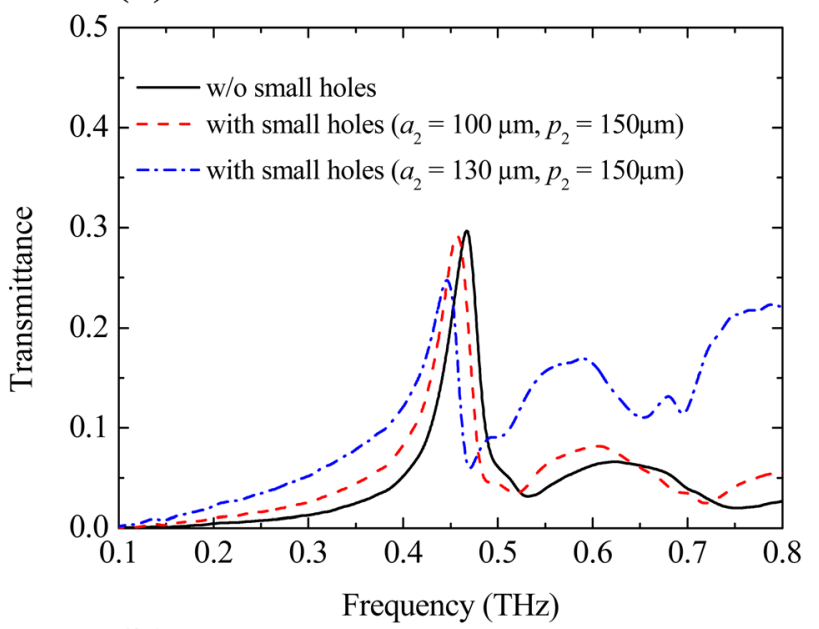

(b)

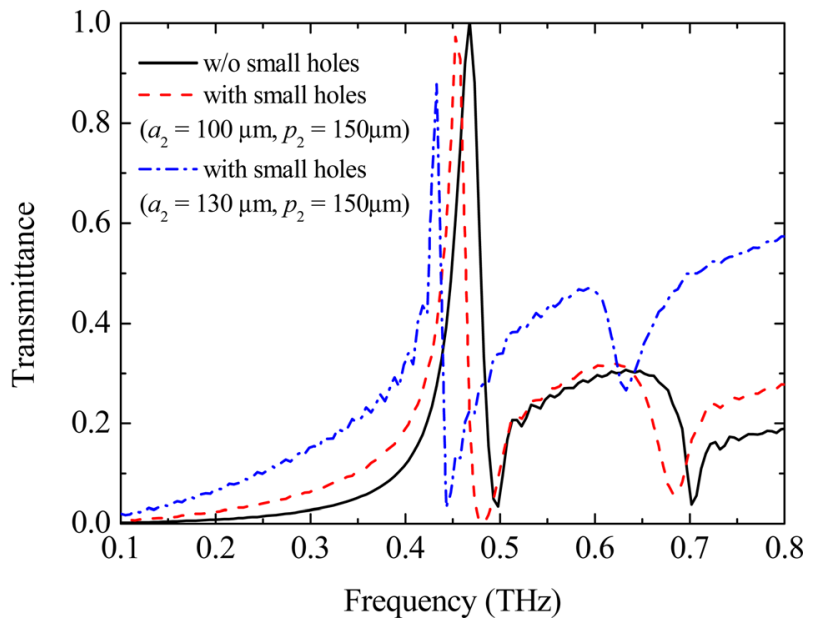

FIG. 2. (Color online) (a) Measured transmission spectra of MHA1 (black solid line), MHA2 (red dashed line), and MHA3 (blue dotted-dashed line). The peak frequency shifts to lower frequency as the size of smaller holes increases. (b) Simulated transmission spectra of metal hole arrays. The geometrical parameters used in this simulation are same to those in experiment. 
in the experiment are smaller, and the bandwidths are relatively broader with respect to those of FDTD calculation.

The shift of the peak frequency is attributed to the modulation of the characteristics of surface waves by analogy with spoof SPPs. ${ }^{14}$ To verify this assumption, we calculated the dispersion relation of the surface waves excited on the flat and corrugated surface of the perfect conductor. According to the theory reported by Garcia-Vidal et al., ${ }^{15}$ the relation between the frequency $\omega$ and the wavenumber in the direction parallel to the surface $k_{x}$ can be written as follows:

$$
\begin{aligned}
& \sqrt{k_{x}^{2}-\frac{\omega^{2}}{c^{2}}}=\frac{S_{0}^{4} \frac{\omega^{2}}{c^{2}}}{\sqrt{\frac{\pi^{2}}{a^{2}}-\varepsilon_{h} \frac{\omega^{2}}{c^{2}}}}, \\
& S_{0}=\frac{2 \sqrt{2} a \sin \left(k_{x} a / 2\right)}{\pi p} \frac{k_{x} a / 2}{}
\end{aligned}
$$

where, $a$ and $p$ are the size and periodicity of the holes, respectively. $\varepsilon_{h}$ is a permittivity of the material inside the

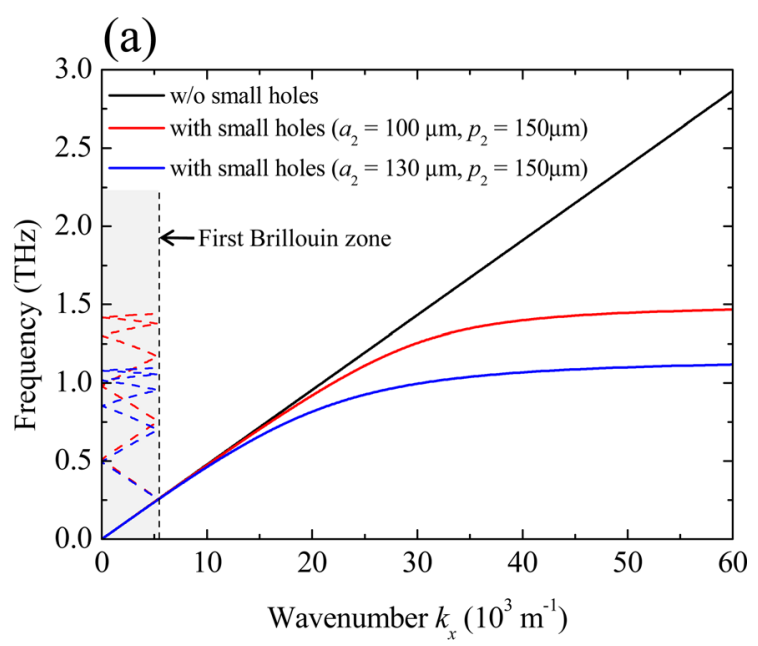

(b)

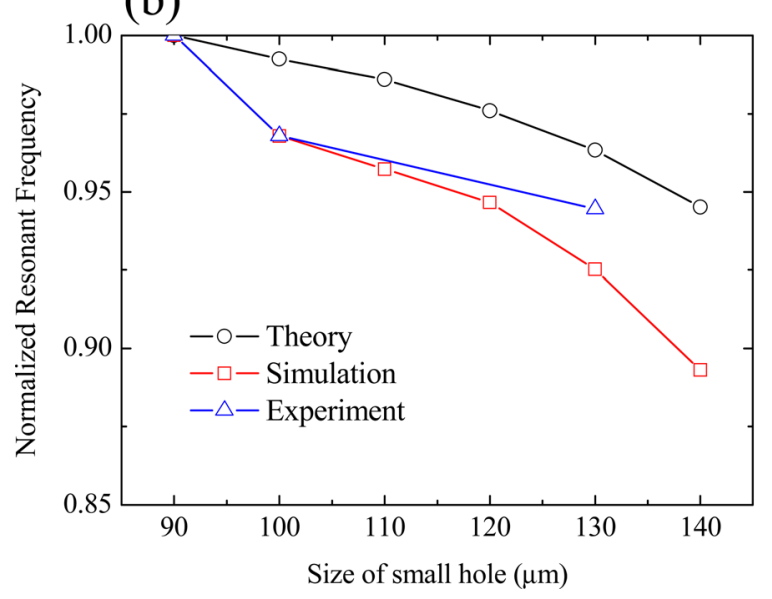

FIG. 3. (Color online) (a) Dispersion relations of spoof surface plasmonpolaritons excited on structured metal surfaces with parameters of MHA1 (black solid line), MHA2 (light gray solid line), and MHA3 (gray solid line). Gray area indicates the first Brillouin zone calculated from the periodicity of $p_{1}=600 \mu \mathrm{m}$. Light gray and gray dashed lines are folded dispersion curves of light gray and gray solid lines, respectively, into the first Brillouin zone. (b) Normalized resonant frequency as a function of the size of small holes. The resonant and peak frequencies are normalized by the value at $a_{2}=90 \mu \mathrm{m}$. These resonant frequencies are calculated or measured by theory (black circle), FDTD simulation (red square), and experiment (blue triangle). holes. Figure 3(a) shows the calculated dispersion relation of the surface waves for a flat surface (black line) and corrugated surface with small hole arrays (light gray and gray lines). In the evaluation of the red and blue lines, we assumed a metal surface that is perforated with a hole array whose geometrical parameters of size and periodicity are the same as those of the samples, i.e., $a_{2}$ and $p_{2}$. On the flat surface, the dispersion curve of the surface wave is almost the same as that of a light line in vacuum, while on the corrugated surface, the dispersion curve bends around $k_{x}=25 \times 10^{3} \mathrm{~m}^{-1}$ and tends to be saturated up to a few $\mathrm{THz}$ with increasing wavenumber. From these results, we conclude that the spoof SPP can be excited on the corrugated metal surface in the $\mathrm{THz}$ region.

By introducing the larger-sized hole array into the aforementioned small hole array, the dispersion curves of Fig. 3 (a) are folded into the first Brillouin zone. Thus, these dispersion curves are now able to interact with the electromagnetic plane waves that come from outside. For a normal incidence as in the experiment, we can estimate the frequency where the dispersion curve reaches $k_{x}=0$, as a resonant frequency of the spoof SPPs. Figure 3(b) shows the resonant frequencies as a function of the size of the small holes estimated from the theory (black circle and line). For comparison, we also plotted the transmission peak frequencies that are observed in the experiment (blue triangle and line) and the simulation (red square and line). The resonant and peak frequencies are normalized by the value at $a_{2}=90 \mu \mathrm{m}$. Excellent agreement among the theory, simulation, and experiment results is observed. This indicates that the characteristics of the surface waves excited on the nearly perfect conductor surface can be modulated by small hole arrays by analogy with spoof surface plasmons.

In summary, we have demonstrated the control of the spectral characteristics of the extraordinary transmissions of metal hole arrays by analogy with spoof SPPs. By introducing the sub-wavelength holes that surround the larger hole arrays, the dispersion curve of the surface waves excited on the structured metal surface can be modified, and consequently, the extraordinary transmissions peak will show the redshift characteristic. Our demonstration indicates that the effective permittivity of metals can be modified and controlled by sub-wavelength hole structures even in the frequency region where metals are regarded as perfect conductors. We believe that our results are promising for producing useful plasmonic devices and metamaterials acting in the $\mathrm{THz}$ region in the near future.

This work was partially supported by the Ministry of Education, Science, Sports, and Culture, Grant-in-Aid for Scientific Research (A) No. 20244047, Grant-in-Aid for Scientific Research on Innovative Areas No. 22109003, Grantin-Aid for Young Scientists (B) No. 21760036, Izumi Science and Technology Foundation, and The Sumitomo Foundation.

${ }^{1}$ T. W. Ebbesen, H. J. Lezec, H. F. Ghaemi, T. Thio, and P. A. Wolff, Nature 391(6668), 667 (1998).

${ }^{2}$ M. Sarrazin and J.-P. Vigneron, Phys. Rev. B 71, 075404 (2005).

${ }^{3}$ F. J. García de Abajo, Rev. Mod. Phys. 79(4), 1267 (2007).

${ }^{4}$ F. Miyamaru and M. Hangyo, Appl. Phys. Lett. 84(15), 2742 (2004). 
${ }^{5}$ J. Bravo-Abad, F. J. García-Vidal, and L. Martín-Moreno, Phys. Rev. Lett. 93(22), 227401 (2004).

${ }^{6}$ F. J. García-Vidal, E. Moreno, J. A. Porto, and L. Martín-Moreno, Phys. Rev. Lett. 95(10), 103901 (2005).

${ }^{7}$ F. Miyamaru and M. Takeda, Phys. Rev. B 79(15), 153405 (2009).

${ }^{8}$ R. Ulrich, Infrared Phys. 7, 37 (1967).

${ }^{9}$ R. Ulrich and M. Tacke, Appl. Phys. Lett. 22, 251 (1973).

${ }^{10}$ P. Lalanne and J. P. Hugonin, Nat. Phys. 2(8), 551 (2006).

${ }^{11}$ L. Aigouy, P. Lalanne, J. P. Hugonin, G. Julié, V. Mathet, and M. Mortier, Phys. Rev. Lett. 98(15), 153902 (2007).
${ }^{12}$ H. Liu and P. Lalanne, Nature 452(7188), 728 (2008).

${ }^{13}$ F. Miyamaru, M. Kamijyo, K. Takano, M. Hangyo, H. Miyazaki, and M. W. Takeda, Opt. Express 18, 17576 (2010).

${ }^{14}$ J. B. Pendry, L. Martín-Moreno, and F. J. Garcia-Vidal, Science 305(5685), 847 (2004).

${ }^{15}$ F. J. Garcia-Vidal, L. Martín-Moreno, and J. B. Pendry, J. Opt. A, Pure Appl. Opt. 7(2), S97 (2005).

${ }^{16}$ A. P. Hibbins, B. R. Evans, and J. R. Sambles, Science 308, 670 (2005).

${ }^{17}$ F. Miyamaru, Y. Saito, K. Yamamoto, T. Furuya, S. Nishizawa, and M. Tani, Appl. Phys. Lett. 96, 211104 (2010). 